Meta

Journal des traducteurs

Translators' Journal

\title{
Terminologie de l'informatique
}

\section{A.-G. Ferre}

Volume 33, numéro 3, septembre 1988

URI : https://id.erudit.org/iderudit/002741ar

DOI : https://doi.org/10.7202/002741ar

Aller au sommaire du numéro

Éditeur(s)

Les Presses de l'Université de Montréal

ISSN

0026-0452 (imprimé)

1492-1421 (numérique)

Découvrir la revue

Citer cet article

Ferre, A.-G. (1988). Terminologie de l'informatique. Meta, 33(3), 427-438.

https://doi.org/10.7202/002741ar

Ce document est protégé par la loi sur le droit d'auteur. L'utilisation des services d'Érudit (y compris la reproduction) est assujettie à sa politique d'utilisation que vous pouvez consulter en ligne.

https://apropos.erudit.org/fr/usagers/politique-dutilisation/
Cet article est diffusé et préservé par Érudit.

Érudit est un consortium interuniversitaire sans but lucratif composé de l’Université de Montréal, l'Université Laval et l'Université du Québec à Montréal. Il a pour mission la promotion et la valorisation de la recherche. https://www.erudit.org/fr/ 


\section{TERMINOLOGIE DE L'INFORMATIQUE}

Au moment où l'informatisation de la société s'étend à tous les domaines, y compris ceux de la vie sociale et de l'information, un vocabulaire cohérent doit être défini pour faciliter les communications entre les professions de l'informatique et les utilisateurs. La terminologie de l'informatique est en fait une création continue à laquelle doivent participer en permanence les industriels, les sociétés de service en ingénierie informatique, les chercheurs, les médias, les linguistes, les enseignants, etc.

Le ministre de l'Industrie, des P. et T. et du Tourisme et le ministre de l'Éducation nationale ont approuvé une nouvelle liste d'expressions et de termes du vocabulaire informatique complétant les arrêtés des 22 décembre 1981 et 30 décembre 1983.

Le présent document résulte des travaux de la Commission de terminologie de l'informatique créée auprès du ministre de l'Industrie, des $P$. et $T$. et du Tourisme. Commission dont la présidence a été confiée au général (CR) André-Georges Ferre.

L'enrichissement du vocabulaire de l'informatique tel qu'il a été conçu à l'échelon national devrait apporter dans les organismes internationaux, où le problème de traduction technique se pose constamment, une suite de références officielles.

L'intérêt porté à cette action par les divers organismes gouvernementaux attachés à ce que le français puisse rester une langue internationale et scientifique susceptible d'exprimer les réalités du monde moderne, doit faire prendre conscience à chacun des efforts à poursuivre et des contributions qu'il est en mesure d'y apporter. Dans ce but, l'AFNOR (Association française de normalisation) a été chargée de donner la plus large diffusion possible aux résultats de l'informatique.

Rappelons que ces termes doivent être obligatoirement utilisés par les organismes publics français ainsi que dans les correspondances qui leur sont adressées. Ces termes seront également utilisés dans tous les ouvrages d'enseignement ou de recherche utilisés dans tous les établissements ou organismes dépendant de l'État. 
Accès direct, n.m.

Mode d'écriture ou de lecture de données se faisant au moyen d'adresses qui repèrent leur emplacement. Anglais : direct access, random access

\section{Accès séquentiel, n.m.}

Mode d'écriture ou de lecture de données effectué en suivant un ordre préétabli de rangement.

Anglais : serial access

Antémémoire, n.f.

Mémoire très rapide destinée à accélérer l'accès aux données les plus fréquemment utilisées.

Anglais : cache memory, cache storage

D'arrière-plan, loc. adv.

Qualifie un traitement qui est exécuté lorsque les ressources ne sont pas affectées à un programme en temps réel ou à des programmes prioritaires.

Anglais : background

Article, n.m.

Groupe de données associées, constituant une unité élémentaire. Synonyme : enregistrement logique.

Autonome, adj.

Se dit d'un matériel lorsqu'il fonctionne indépendamment de tout autre.

Anglais : off line

\section{Banque de données, n.f.}

Ensemble de données relatif à un domaine défini des connaissances et organisé pour être offert aux consultations d'utilisateurs.

Anglais : data bank

Base de données, n.f.

Ensemble de données organisé en vue de son utilisation par des programmes correspondant à des applications distinctes et de manière à faciliter l'évolution des données et des programmes.

Anglais : data base

Bit, n.m.

Information représentée par un symbole à deux valeurs généralement notées 0 et 1 , associées aux deux états d'un dispositif.

Anglais : bit

Bogue, n.f.

Défaut de conception ou de réalisation se manifestant par des anomalies de fonctionnement.

Anglais : bug

Boule de commande, n.f.

Boule qui, par rotation dans son logement, permet de déplacer sur l'écran d'une visu un curseur et d'en relever à volonté les coordonnées.

Anglais : trackball, rolling ball 


\section{Bureautique, n.f.}

Ensemble des techniques et des moyens tendant à automatiser les activités de bureau et principalement le traitement et la communication de la parole, de l'écrit et de l'image.

Bus, n.m.

Dispositif non bouclé destiné à assurer simultanément les transferts d'information entre différents sousensembles d'un système informatique selon des spécifications physiques et logiques communes.

Anglais : bus

Calculette, n.f.

Calculatrice électronique de petite dimension.

Clicher, v.

Recopier le contenu, à un instant déterminé, de tout ou partie d'une mémoire sur un autre support.

Note : Par extension, le nom correspondant pourrait être cliché ou cliché-mémoire.

Anglais : to dump

Codet, n.m.

Groupe d'éléments représentant, selon un code, une donnée élémentaire.

Note : "Codet » est destiné à remplacer des expressions telles que " combinaison de code » ou " mot de code". Le terme " code " désigne en effet l'ensemble des règles de représentation des données élémentaires par des coclets. Par exemple, selon le code Morse, les caractères et différentes fonctions sont représentés par des codets qui sont des groupes de points et de traits.

Anglais : code element

\section{Compatibilité, n.f.}

Qualité d'un matériel ou d'un logiciel conforme aux règles d'interface d'un système informatique défini, et clont l'introduction n'altère pas les conditions de fonctionnement de ce système.

Anglais : compatibility

\section{Courtier, n.m.}

Intermédiaire entre serveurs et utilisateurs, assistant ces derniers dans le choix et la consultation des banques de données.

Anglais : broker

Déboguer, v.tr.

Éliminer les bogues.

Terme dérivé : DÉBOGAGE

Anglais : to debug

Défilement, n.m.

Déplacement vertical ou horizontal du contenu d'un écran de visualisation à l'intérieur d'une fenêtre de telle façon que de nouvelles données apparaissent à un bord alors que d'autres disparaissent au bord opposé. Anglais : scrolling

\section{Descripteur, n.m.}

Mot ou locution contribuant à caractériser l'information contenue dans un document et à en faciliter la recherche. 
Dévideur, n.m.

Dérouleur de bande magnétique voué par construction à la création en continu de sauvegardes des informations contenues dans un disque.

Anglais : streamer

Didacticiel, n.m.

Logiciel spécialisé pour l'enseignement.

Disque magnétique, n.m.

DISQUE (par abréviation)

Disque recouvert d'une couche magnétique où sont enregistrées des données.

Anglais : magnetic disk

Disque optique, n.m.

Disque où sont enregistrées des données lisibles par un procédé optique.

Disquette, n.f.

Disque magnétique souple, de dimension et de capacité réduites.

Anglais : diskette, floppy disk

Donnée, n.f.

Représentation d'une information sous une forme conventionnelle destinée à faciliter son traitement. Anglais : data

Écran tactile, n.m.

Écran muni d'un dispositif qui, sur une visu, permet l'entrée de données en désignant du doigt des zones d'écran.

Anglais : touch screen

Éditeur, n.m.

Programme qui permet à partir d'une visu d'introduire des données textuelles ou graphiques ou d'en modifier la disposition.

Anglais : editor

Format, n.m.

- Agencement structuré d'un support de données.

- Disposition des données elles-mêmes.

Termes dérivés : FORMATER v.tr., FORMATAGE n.m.

Fusionner, v.

Réunir en un seul ensemble les éléments de plusieurs ensembles rangés suivant les mêmes critères. Anglais : to merge

Génie informatique, n.m.

Conception, réalisation et validation des systèmes informatiques.

Génie logiciel, n.m.

Ensemble des activités de conception et de mise en cuvre des produits et des procédures tendant à rationaliser la production du logiciel et son suivi.

Anglais : software engineering 
Grapheur, n.m.

Programme ou outil permettant de représenter des tableaux de résultats sous forme de graphiques.

Incrément, n.m.

Quantité dont on augmente la valeur d'une variable à chaque phase de l'exécution d'un programme.

Termes dérivés : INCRÉMENTER v.tr., INCRÉMENTIEL adj., DÉCRÉMENT n.m.

Anglais : increment

Infographie, n.f.

Application de l'informatique à la représentation graphique et au traitement de l'image.

Information, n.f.

Élément de connaissance susceptible d'être représenté à l'aide de conventions pour être conservé, traité ou communiqué.

Informatique, adj.

Qui se rapporte à l'informatique.

Informatique, n.f.

Science du traitement rationnel, notamment par machines automatiques, de l'information considérée comme le support des connaissances humaines et des communications dans les domaines technique, économique et social (définition approuvée par l'Académie française).

Anglais : electronic data processing

Instaurer, v.tr.

Mettre dans un état actif.

Anglais : to set

Instruction, n.f.

Consigne exprimée dans un langage de programmation.

Anglais : instruction, statement

Interactif, adj.

Qualifie les matériels, les programmes ou les conditions d'exploitation qui permettent des actions réciproques en mode dialogué avec des utilisateurs ou en temps réel avec des appareils.

Anglais : interactive

Interface, n.f.

Jonction entre deux matériels ou logiciels leur permettant d'échanger des informations par l'adoption de règles communes physiques ou logiques.

Anglais : interface

Invite, n.f.

Message visuel ou sonore sollicitant conformément à une disposition programmée l'avis ou l'action de l'opérateur.

A.nglais : prompt

Langage formel, n.m.

Langage qui utilise un ensemble de termes et de règles syntaxiques pour permettre de communiquer sans aucune ambiguïté (par opposition à langage naturel). 
En ligne, loc. adj.

Se dit d'un matériel lorsqu'il fonctionne en relation directe avec un autre.

Anglais : on line

Listage, n.m.

Document en continu produit par une imprimante d'ordinateur. Action de lister.

Anglais : listing

Lister, v.tr.

Produire un document en continu à l'aide d'une imprimante d'ordinateur. Présenter des données ou des instructions.

Anglais : to list

\section{Logiciel, n.m.}

Ensemble des programmes, procédés et règles, et éventuellement de la documentation, relatifs au fonctionnement d'un ensemble de traitement de l'information.

Anglais : software

Ludiciel, n.m.

Logiciel de jeu.

Maintenance, n.f.

Ensemble d'actions tendant à prévenir ou à corriger les dégradations d'un matériel afin de maintenir ou de rétablir sa conformité aux spécifications.

Note : Ce terme ne doit pas être employé pour désigner les améliorations fonctionnelles ou de performances apportées à chaque nouvelle version d'un logiciel.

Manche à balai, n.m.

Dispositif de commande à plusieurs degrés de liberté servant à déplacer le curseur d'une visu. Abréviation : manche (n.m.).

Synonyme : poignée (n.f.).

Mappe, n.f.

Représentation de la localisation d'ensembles de données en vue d'en faciliter l'accès.

Termes dérivés : MAPPER v.tr., MAPPAGE n.m.

Anglais : map

Marquage, n.m.

Action de faire ressortir un élément graphique ou une partie de texte par modification de ses attributs visuels. Anglais : highlighting

Matériel, n.m.

Ensemble des éléments physiques employés pour le traitement des données.

Anglais : hardware

Mémoire, n.f.

Organe qui permet l'enregistrement, la conservation et la restitution des données.

Anglais : storage, memory 
Mémoire de masse, n.f.

Mémoire externe de très grande capacité.

Anglais : mass storage

Mémoire morte, n.f.

Mémoire dont le contenu ne peut être modifié en usage normal.

Anglais : read only memory, ROM

Mémoire tampon, n.f.

Mémoire ou partie de mémoire permettant le stockage temporaire de données entre deux organes ayant des caractéristiques différentes.

Abréviation : tampon (n.m.)

Anglais : buffer

Mémoire vive, n.f.

Mémoire dont le contenu peut être modifié en usage normal.

Anglais : $R A M$

Messagerie électronique, n.f.

Abréviation : MESSAGERIE n.f.

Service géré par ordinateur fournissant aux utilisateurs habilités les fonctions de saisie, de distribution et de consultation différée de messages notamment écrits, graphiques ou sonores.

Anglais : message handling, electronic mail

Microprocesseur, n.m.

Processeur miniaturisé dont tous les éléments sont, en principe, rassemblés en un seul circuit intégré. Anglais : microprocessor

Microprogramme, n.m.

Ensemble ordonné d'instructions enregistrées dans une mémoire interne fonctionnellement distincte de la mémoire principale, et dont l'exécution permet celle d'une instruction non câblée du langage machine, ou d'une fonction programmée.

Anglais : firmware

Mode dialogué, n.m.

Mode de traitement de données permettant un dialogue entre système informatique et utilisateur.

Anglais : conversational mode

Mot clé, n.m.

Descripteur extrait du texte qu'il caractérise ou d'un thésaurus.

Anglais : keyword

Multiprogrammation, n.f.

Technique d'exploitation permettant l'exécution imbriquée de plusieurs programmes menés de front. Anglais : multiprogramming

Multitraitement, n.m.

Mode de fonctionnement d'un ordinateur selon lequel plusieurs processeurs ayant accès à des mémoires comrnunes peuvent opérer en parallèle sur des programmes différents.

Anglais : multiprocessing 
Numérique, adj.

Se dit, par opposition analogique, de la représentation de données ou de grandeurs physiques au moyen de caractères - des chiffres généralement - et aussi des systèmes, dispositifs ou procédés employant ce mode de représentation discrète.

Anglais : digital, numerical, numeric

Numériser, $v$.

Représenter un signal (caractère, image, impulsion, etc.) sous forme numérique.

Termes dérivés : NUMÉRISEUR, NUMÉRISATION

Anglais : to digitize

Ordinateur, n.m.

Équipement informatique de traitement automatique de données comprenant les organes nécessaires à son fonctionnement autonome.

Anglais : computer

Ordinateur individuel, n.m.

Ordinateur d'usage individuel, dans un cadre professionnel ou domestique.

Anglais : personal computer

Partage de temps, n.m.

Technique d'exploitation d'un même ordinateur par plusieurs utilisateurs qui exécutent simultanément en mode dialogué, chacun à son propre rythme, des travaux indépendants.

Remarque : dans l'usage courant on peut utiliser l'expression "travail en temps partagé ".

Anglais : time-sharing

Photostyle, n.m.

Dispositif d'entrée que l'opérateur pointe directement sur l'écran d'une visu.

Anglais : light pen

Pixel, n.m.

Plus petit élément d'une présentation visualisée auquel peuvent être affectés séparément des attributs tels que luminosité, couleur, clignotement.

Anglais : pixel

Pointeur, n.m.

Donnée permettant de retrouver l'adresse d'un groupe de données.

Anglais : pointer

Portabilité, n.f.

Aptitude d'un programme à être utilisé sur des sytèmes informatiques de types différents.

Processeur, n.m.

Organe destiné, dans un ordinateur ou une autre machine, à interpréter et exécuter des instructions. Par analogie, ensemble de programmes permettant d'exécuter sur un ordinateur des programmes écrits dans un certain langage.

Termes dérivés : BI-PROCESSEUR n.m., ordinateur ayant 2 processeurs centraux ; TRI-PROCESSEUR n.m., ordinateur ayant 3 processeurs centraux; MULTIPROCESSEUR n.m., ordinateur ayant plusieurs processeurs centraux.

Anglais : processor 


\section{Processeur vectoriel, n.m.}

Processeur conçu pour appliquer simultanément le même traitement à des éléments homologues de tableaux de données.

Anglais : array processor

Producteur, n.m.

Dans le domaine des banques de données, désigne celui qui les crée et les met à jour.

Note : Le terme prestataire, incorrect dans ce sens, est à proscrire.

Progiciel, n.m.

Ensemble complet et documenté de programmes conçu pour être fourni à plusieurs utilisateurs, en vue d'une même application ou d'une même fonction.

Anglais : package

Relancer, v.tr.

Remettre en marche un système informatique après sa restauration

Anglais : to restart

Répertoire, n.m.

Liste d'identificateurs, classés selon des arguments appropriés, permettant l'accès aux informations qu'ils désignent.

Anglais : directory

Requête, n.f.

Expression formalisée d'une demande.

Anglais : request

Réseau local, n.m.

Ensemble connexe, à caractère privatif, de moyens de communication établi sur un site restreint, pourvu de règles de gestion du trafic et permettant des échanges internes d'informations de toute nature notamment sous forme de données, sons, images, etc.

Note : Le réseau local, ainsi défini en informatique, ne doit pas être confondu avec la notion de "réseau local de raccordement ", utilisée dans les télécommunications.

Anglais : local area network

Restaurer, v.tr.

Fiemettre dans un état de référence.

Anglais : to reset, to restore.

Résumé, n.m.

Fiédaction abrégée reprenant l'essentiel d'un texte.

Anglais : abstract

Révision, n.f.

Logiciel comportant des corrections par rapport à l'état précédent. Anglais : release

\section{Robotique, n.f.}

Ensemble des études et des techniques de conception et de mise en cuvre des robots effectuant des tâches déterminées en s'adaptant à leur environnement. 
De secours, loc. adj.

Qualifie les procédures et les matériels destinés à être utilisés dans certains cas d'anomalie de fonctionnement. Anglais : back up

\section{Serveur, n.m.}

Organisme exploitant un système informatique permettant à un demandeur la consultation et l'utilisation directe d'une ou plusieurs banques de données.

Par extension, le système informatique lui-même.

Anglais : on line data service

Souris, n.f.

Disposition auxiliaire d'une visu qui, par mouvement sur une surface, permet de déplacer sur l'écran un curseur et d'en relever à volonté les coordonnées.

Anglais : mouse

Spoule, n.m.

Mode d'exploitation d'un ordinateur en multiprogrammation selon lequel les opérations d'entrée et de sortie sont automatiquement dissociées de traitements intermédiaires, les données correspondantes étant placées dans des mémoires tampons.

Anglais : spool

\section{Surbrillance, n.f.}

Marquage par une luminosité plus grande.

Anglais : brightening

Survol, n.m.

Exploration rapide sur visu, sans possibilité de modification, du contenu d'une mémoire.

Anglais : browsing

\section{Système d'exploitation, n.m.}

Logiciel gérant un ordinateur, indépendant des programmes d'application mais indispensable à leur mise en ceuvre.

Anglais : operating system

Système de gestion de bases de données (S.S.G.B.), n.m.

Pour une base de données, logiciel permettant d'introduire les données, de les mettre à jour et d'y accéder. Anglais : Data Base Mangement System, D.B.M.S.

Tableur, n.m.

Programme de création et de manipulation interactives de tableaux numériques visualisés. Anglais : spreadsheet

Télématique, n.f.

Ensemble des services de nature ou d'origine informatique pouvant être fournis à travers un réseau de télécommunications.

Télématique, n.m.

Exploitation automatisée de systèmes informatiques utilisant des réscaux de télécommunications. 


\section{Télétraitement par lot, n.m.}

Télétraitement qui comporte un groupement par lots des programmes à exécuter ou des données à traiter.

Anglais : remote batch teleprocessing

Temps réel, n.m.

Mode de traitement qui permet l'admission des données à un instant quelconque et l'obtention immédiate des résultats.

Anglais : real time

Terminal, n.m.

Appareil permettant l'accès à distance à un système informatique.

Anglais : terminal

Test de performance, n.m.

Evaluation des performances, d'un système par simulation des conditions réelles d'utilisation, à l'aide de programmes échantillons.

Anglais : benchmark

En texte intégral, loc. adj.

Qualifie une banque de données dans laquelle sont enregistrés des documents complets et non des résumés ou cles descripteurs.

Anglais : full text

Tirage, n.m. ou Fac-Sim, n.m.

Document graphique résultant du transfert sur un support permanent d'une image présentée sur une visu. Anglais : hard copy

Traitement automatique des données, n.m.

Ensemble des opérations réalisées par des moyens automatiques relatif à la collecte, l'enregistrement, l'élaboration, la modification, la conservation, la destruction, l'édition de données et d'une façon générale leur exploitation.

Remarque : Dans l'usage courant, l'expression « traitement automatique de l'information » est également employée.

Anglais : A.D.P.

Traitement de texte, n.m.

Ensemble des opérations de création, manipulation et impression de texte effectuées à l'aide de moyens électroniques.

Anglais : word processing, text processing

Traitement par lots, n.m.

Mode de traitement des données suivant lequel les programmes à exécuter ou les données à traiter sont groupées en lots.

Anglais : batch processing

Transporteur, n.m.

Organisme gérant un réseau de télécommunications par lequel des utilisateurs accèdent aux centres-serveurs. 
Tutoriel, n.m.

Guide de présentation et d'initiation à l'utilisation d'un ensemble de notions, d'un logiciel, d'un matériel, etc. Anglais : tutorial

\section{Version, n.f.}

Logiciel contenant de nouvelles fonctions modifiant un logiciel ancien.

Anglais : release, version

Visu, n.f. Visuel, n.m.

Appareil permettant la présentation visuelle et non permanente d'informations.

Anglais : display device

Visualiser, v.tr.

Inscrire les résultats d'un traitement sur une visu.

Anglais : to display

SECRÉTARIAT DE LA COMMISSION MINISTÉRIELLE DE TERMINOLOGIE DE

L'INFORMATIQUE

Paris, France 